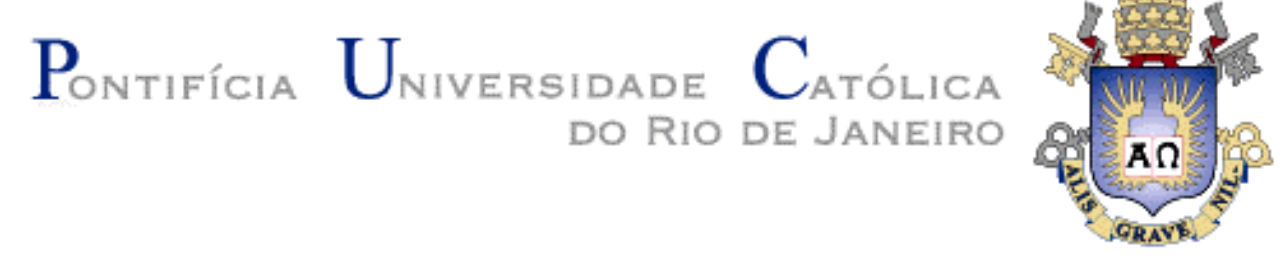

Beatriz Silva Amaral

\title{
CARACTERIZAÇÃO QUÍMICA DE MATERIAL PARTICULADO (PM10) COLETADO EM PONTOS ESTRATÉGICOS DA REGIÃO METROPOLITANA DO RIO DE JANEIRO
}

Dissertação apresentada como requisito parcial para obtenção do título de Mestre pelo programa de Pós-Graduação em Química da PUC-Rio.

Orientadora: Prof ${ }^{\mathrm{a}}$. Adriana Gioda Co-orientadora: Prof ${ }^{\mathrm{a}}$. Tatiana Dillenburg Saint'Pierre 


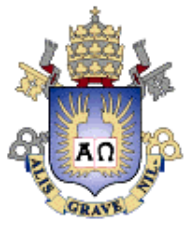

Beatriz Silva Amaral

\title{
CARACTERIZAÇÃO QUÍMICA DE MATERIAL PARTICULADO (PM10) COLETADO EM PONTOS ESTRATÉGICOS DA REGIÃO METROPOLITANA DO RIO DE JANEIRO
}

Dissertação apresentada como requisito parcial para obtenção do título de Mestre pelo programa de Pós-Graduação em Química da PUC-Rio. Aprovada pela Comissão Examinadora abaixo assinada.

\author{
Prof ${ }^{a}$. Adriana Gioda \\ Orientadora \\ Departamento de Química - PUC - Rio \\ Prof $^{a}$. Tatiana Dillenburg Saint'Pierre \\ Co-Orientadora \\ Departamento de Química - PUC - Rio \\ Prof $^{\mathrm{a}}$. Neusa Pereira Arruda \\ IFRJ - Rio de Janeiro \\ Prof $^{a}$. Maria da Conceição Vanderley Ramos \\ UFRJ - Rio de Janeiro \\ Prof. Otávio Versiane Cabral \\ IFRJ - Rio de Janeiro \\ Prof. José Eugenio Leal \\ Coordenador Setorial do Centro \\ Técnico Científico - PUC-Rio
}

Rio de Janeiro, 24 de Setembro de 2010 
Todos os direitos reservados. É proibida a reprodução total ou parcial do trabalho sem autorização da universidade, da autora e do orientador.

\section{Beatriz Silva Amaral}

Graduou-se em Licenciatura em Química pela Universidade do Grande Rio (UNIGRANRIO) 2001, ingressou na rede Estadual de Educação em 2005 e no quadro técnico do Instituto Federal do Rio de Janeiro em 2008.

Ficha Catalográfica

\section{Amaral, Beatriz S.}

Caracterização Química de Material Particulado (PM10) Coletado em Pontos Estratégicos da Região Metropolitana do Rio de Janeiro / Beatriz Silva Amaral; orientadora: Adriana Gioda - 2010.

140 f. : il.(col.) ; $30 \mathrm{~cm}$

Dissertação (Mestrado em Química)-Pontifícia Universidade Católica do Rio de Janeiro, Rio de Janeiro, 2010.

Inclui bibliografia

1. Química - Teses. 2. Material particulado. 3. metais. 4. Fração aquosa. 5. transporte de longa distância. I. Gioda, Adriana. II. Amaral, Beatriz Silva. III. Pontifícia Universidade Católica do Rio de Janeiro. Departamento de Química. IV. Título.

CDD: 540 
Dedico este trabalho a Deus, fonte de todo o conhecimento, princípio e fim, Quem tudo pode dar e tudo pode tirar. Aos meus mestres incondicionais, meus pais José e Constância, pelo carinho, amor e sacrifício por tudo que fizeram para eu chegar até aqui. Amo vocês demais. 


\section{Agradecimentos}

À minha família, meu esposo Adriano, pela paciência e seu carinho, nem de mais e nem de menos, mas na medida certa e minhas filhas Ariane e Ana, por suportar os momentos de ausência e total estresse;

Aos meus irmãos Raulino, Norma e Fabiana, por acreditarem em mim, por me dar muita força em todos os meus projetos. Amo vocês.

A minha Orientadora professora Adriana Gioda, por confiar em mim na realização deste trabalho pela sua ajuda na medida certa.

A professora Tatiana, pelo acolhimento e por ter facilitado o uso do laboratório.

A todo o Departamento de Química, principalmente à Fátima, por estar sempre atenta a datas e prazos e por manter todos informados.

Aos amigos conquistados no decorrer deste período, que faço questão de mencionar aqui porque foram de extrema importância:

Catarina, Carol, Soraya, Ricardo, Flávia, Eider, Jefferson, Fernanda e outros;

À Cibele, pela disponibilidade, carinho e amizade na grande ajuda na parte estatística. 
À Isabela Luizi, aluna de iniciação científica que ajudou nas análises;

A todos os técnicos que trabalham no Labspectro, Verônica, Álvaro, Rafael, André, Maurício e Danielle, que me ajudaram nas análises.

Aos amigos que me ajudaram em oração da Comunidade São Vicente de Paulo;

Aos professores Hiran e Tadeu por confiar e assinar a carta de recomendação;

Ao diretor da IFRJ da unidade Maracanã por facilitar no que fosse possível;

A instituição PUC-Rio por financiar minha pesquisa.

Ao INEA por coletar e fornecer as amostras e os dados necessários.

A instituição que trabalho EE Lélia Gonzáles, por compreender as faltas e atrasos durante este período;

Aos professores da EE Lélia Gonzáles, pela torcida;

E agradeço a todos que indiretamente estiveram envolvidos com esta pesquisa e facilitaram de alguma forma para obtenção desses resultados. A vocês, meu muito obrigado.

Professora Roberta Ziolli, pela disponibilização do laboratório e a bióloga Daniele pelas análises de TOC. 


\section{Resumo}

Amaral, Beatriz S; Gioda, Adriana. Caracterização Química de Material Particulado (PM10) Coletado em Pontos Estratégicos da Região Metropolitana do Rio de Janeiro. Rio de Janeiro, 2010. 140p. Dissertação de Mestrado - Departamento de Química, Pontifícia Universidade Católica do Rio de Janeiro.

O objetivo deste estudo foi determinar a concentração de metais, compostos orgânicos e inorgânicos por suspensão aquosa e análise dos metais por abertura ácida presentes nas amostras de PM10 coletadas em áreas urbanas, industrial e rural da Região Metropolitana do Rio de Janeiro. A concentração de PM10 foi determinada por análise gravimétrica. Espécies inorgânicas e orgânicas presentes nos extratos aquosos foram determinadas por cromatografia de íons e carbono orgânico total (TOC). Os extratos ácidos, e também os aquosos, foram analisados por ICP-MS (espectrometria de massa com plasma indutivamente acoplado) e por ICP OES (espectrometria de emissão óptica com plasma indutivamente acoplado) para determinar a concentração de metais. As correlações entre as concentrações de PM10 e de metais foram avaliadas, considerando os dados meteorológicos para cada ponto de coleta e origem das massas de ar. Os resultados mostraram que as concentrações médias de PM10 foram de $36 \mu \mathrm{g} \mathrm{m}^{-3}$ na área rural, $50 \mu \mathrm{g} \mathrm{m}^{-3}$ na área urbana e $74 \mu \mathrm{g}$ $\mathrm{m}^{-3}$ na área industrial. A concentração de PM10 medida na área industrial ultrapassou, em geral, o limite estabelecido pela resolução CONAMA, de $50 \mu \mathrm{g} \mathrm{m}^{-3}$. O teor de metais apresentou maior concentração na área industrial, sendo predominantes ferro, zinco, alumínio, titânio, manganês, cromo, níquel, cádmio e chumbo. Na área de maior tráfego, foi detectada a maior concentração de cobre e vanádio. As espécies iônicas foram maiores na área urbana, devido à proximidade do oceano. Na área rural, menores concentrações de espécies antropogênicas, como sulfato, nitrato e alguns metais ( $\mathrm{Pb}, \mathrm{Cr}, \mathrm{V}$ e $\mathrm{Ni}$ ) foram medidas. Assim, as 
concentrações das espécies estudadas são influenciadas pelas características locais. Porém, também foi observado a influencia do transporte de poluentes de longa distância, como queimadas e poeiras dos desertos do continente africano. Os percentuais dos extratos ácido/aquoso demonstraram que os metais extraídos de forma mais eficiente foram $\mathrm{V}$ e $\mathrm{Ni}$ ( $>45 \%$ ), enquanto que os menos extraídos foram $\mathrm{Al}$ e $\mathrm{Fe}(<3 \%)$, conforme o esperado. A fração aquosa é importante para avaliar a biodisponibilidade de metais associados a danos à saúde.

\section{Palavras Chaves}

PM10; metais; fração aquosa; fração ácida; transporte de longa distância; carbono orgânico solúvel e íons solúveis. 


\section{Abstract}

Amaral, Beatriz S; Gioda, Adriana (Advisor) Chemical Characterization of Particulate Matter (PM10) Collected in Strategic Points in the Metropolitan Region of Rio de Janeiro. Rio de Janeiro, 2010. 140p. MSc. Dissertation - Departamento de Química, Pontifícia Universidade Católica do Rio de Janeiro.

The scope of this study was to measure the concentration of metals and organic and inorganic anions in aqueous and acid extracts of PM10 samples collected in urban, industrial and rural zones of Rio de Janeiro. PM10 concentration was determined by gravimetric analysis and the organic and inorganic species in the aqueous extracts were determined by ion chromatography and total organic carbon (TOC). The aqueous and acid extracts were analyzed by inductively coupled plasma mass spectrometry (ICP-MS) and by ICP OES (inductively coupled plasma optical emission spectrometry), in order to determine metal content. Correlations between PM10 and metal concentrations were evaluated, considering meteorological data for each site. Results showed that the average PM10 concentrations were $36 \mu^{-3} \mathrm{~m}^{-3} ; 50$ $\mu \mathrm{g} \mathrm{m}^{-3}$ and $74 \mu \mathrm{g} \mathrm{m}^{-3}$ at the rural, downtown and industrial sites, respectively. The measured PM10 concentration in the industrial site exceeded the limit allowed by the Brazilian law, which is $50 \mu \mathrm{g} \mathrm{m} \mathrm{m}^{-3}$. While industrial area had the highest concentrations of metals, such as $\mathrm{Fe}, \mathrm{Zn}, \mathrm{Al}, \mathrm{Ti}, \mathrm{Mn}, \mathrm{Cr}, \mathrm{Ni}, \mathrm{Cd}$ and $\mathrm{Pb}$, the highest concentrations of $\mathrm{Cu}$ and $\mathrm{V}$ were found in areas with higher traffic densities. Ionic species had higher concentrations in the urban site due to the proximity to the ocean. The lowest concentrations of species from anthropogenic sources, such as sulfate, nitrate and some metals $(\mathrm{Pb}, \mathrm{Cr}, \mathrm{V}$ and $\mathrm{Ni}$ ) were measured in the rural area. Hence, studied species concentrations are influenced by site locations, but the influence by the transportation of long distance pollutants, such as biomass burning and dust from African deserts was also observed. The acid/aqueous percentiles showed that the most efficiently extracted metals in the aqueous phase were $\mathrm{V}$ and $\mathrm{Ni}(>45 \%)$ while the less efficiently extracted were $\mathrm{Al}$ and $\mathrm{Fe}(<3 \%)$, as expected. The aqueous fraction is 
important to evaluate the bioavailability of metals that are associated to damage to the human health.

\section{Keywords}

PM10; metals, aqueous fraction; acid fraction, long-distance transport; soluble organic carbon and soluble ions. 


\section{Sumário}

$\begin{array}{ll}\text { 1. Atmosfera } & 24\end{array}$

1.1. Poluição Atmosférica $\quad 25$

1.2. Padrões da qualidade do ar 28

1.3. Material Particulado 31

1.3.1. Origem e Classificação do Material Particulado 31

1.4. Condições meteorológicas e transporte de longa distância 34

1.5. Danos a Saúde e ao Meio Ambiente 35

$\begin{array}{ll}2 \text { Objetivo } & 39\end{array}$

2.1. Objetivo Geral 39

2.2. Objetivos Específicos 39

3 Características das Áreas de Amostragem $\quad 40$

3.1. Município de Seropédica 43

3.2. Município de Duque de Caxias 44

3.3. Centro 45

3.4. Amostragem 46

$\begin{array}{ll}\text { 3.5. Tratamento dos Filtros } & 48\end{array}$

3.5.1. Extração das Espécies Solúveis em Água 48

3.5.2. Extração do Carbono Orgânico Solúvel em Água - COSA 49

3.5.3. Extração Ácida 49 
4.1. Cromatografia de íons 51

4.1.1. Aspectos Gerais 51

4.1.2. Calibração e limites de detecção 52

4.2. Espectrometria de massa com plasma indutivamente acoplado (ICP-MS) 55

4.2.1. Aspectos gerais $\quad 55$

4.2.2 Calibração e limites de detecção e quantificação 57

4.3. Espectrometria de emissão ótica com plasma indutivamente acoplado (ICP OES) 58

4.3.1. Aspectos gerais 58

4.3.2. Calibração e limite de detecção e quantificação 60

4.4. Carbono orgânico solúvel em água (COSA) 61

4.4.1. Calibração limite de detecção e quantificação 62

5. Dados meteorológicos e trajetórias 63

5.1. Dados meteorológicos 63

5.2. Classificação da origem de massas de ar e imagens de satélites 65

6. Análise Estatística 69

$\begin{array}{ll}\text { 7. Resultados e discussões } & 70\end{array}$

7.1. Material Particulado (PM10) 70

7.2. Composição química da fração aquosa de PM10 75

7.2.1. Carbono orgânico total 75 
7.2.2. Espécies iônicas

7.3. Metais $\quad 82$

7.4. Composição química da fração ácida de PM10 83

7.5. Influências da variabilidade temporal $\quad 89$

7.6. Influências das trajetórias das massas de ar 94

7.7. Período Sazonal Úmido e Período Sazonal Seco 95

7.7.1. Período Sazonal Úmido e Período Sazonal Seco

$\begin{array}{ll}\text { região Seropédica } & 96\end{array}$

7.7.2. Período Sazonal Úmido e Período Sazonal Seco região Centro 97

7.7.3. Período Sazonal Úmido e Período Sazonal Seco da

$\begin{array}{lc}\text { Região de Duque de Caxias } & 98\end{array}$

$\begin{array}{ll}\text { 7.8. Análise estatística } & 99\end{array}$

$\begin{array}{ll}\text { 8. Conclusão } & 109\end{array}$

$\begin{array}{ll}\text { 9. Referências Bibliográficas } & 111\end{array}$

$\begin{array}{ll}\text { 10. Anexol } & 117\end{array}$

$\begin{array}{ll}\text { 10.1. Curva do } \mathrm{Cl} & 117\end{array}$

$\begin{array}{ll}\text { 10.2. Curva do ICP-MS } & 119\end{array}$

$\begin{array}{lr}\text { 10.3. Curva ICP OES } & 120\end{array}$

$\begin{array}{ll}\text { 10.4. Curva de IC e TC } & 122\end{array}$

11. Anexo II: Tabelas dos resultados obtidos 123 
12. Anexo III

12.1. Histogramas e gráficos de normalidades obtidos para todas as espécies pelo estudo estatístico 


\section{Lista de figuras}

Figura 1: Representação das camadas que envolvem a Terra com a variação média de temperatura e altitude.

Figura 2: Contribuição de fontes poluidoras da RMRJ

Figura 3: Representação esquemática dos mecanismos de formação do aerossol secundário.

Figura 4: Esquema da distribuição granulométrica das partículas da atmosfera mostrando os três modos, as principais fontes e os principais processos envolvidos na remoção em cada modo.

Figura 5: Grau de penetração das partículas no trato respiratório. 36

Figura 6: Material particulado depositado nas plantas.

Figura 7: Posição geográfica do Município do Rio de Janeiro.

Figura 8: Mapa das áreas prioritárias e áreas que requerem acompanhamento.

Figura 9: Região Metropolitana do Rio de Janeiro com a localização dos pontos de amostragem deste estudo.

Figura 10: Ponto de coleta de Seropédica.

Figura 11: Vista Panorâmica da área próximo ao ponto de coleta em Duque de Caxias.

Figura 12: Foto da Presidente Vargas próximo ao ponto de coleta da região Centro. 
Figura 13: Amostrador de Grande Volume com cabeça

de Separação por Impactação Inercial. $\quad 47$

$\begin{array}{ll}\text { Figura 14: Fotos dos Filtros no laboratório. } & 48\end{array}$

Figura 15: Extração aquosa. $\quad 49$

Figura 16: Extração ácida. $\quad 50$

Figura 17: Cromatógrafo de ĺons usado neste trabalho. 52

Figura 18: Cromatograma da água utilizada no trabalho 53

Figura 19: Cromatograma de uma amostra da região de Seropédica. 53

Figura 20: Esquema do ICP-MS do sistema de introdução de

amostra ao detector. $\quad 55$

Figura 21: ICP OES modelo Optima DV 4300 (PerkinElmer). 59

Figura 22: Temperaturas máximas, médias e mínimas utilizadas para avaliação do ar da RMRJ.

Figura 23: Precipitação acumulada mensal para RMRJ para

o ano 2008.

64

Figura 24: Precipitação acumulada mensal para RMRJ

para o ano 2009.

Figura 25: Trajetória de massas de ar de origem marinha. 66

Figura 26: Trajetória de massas de ar de origem continental. $\quad 67$

Figura 27: Trajetória de massas de ar de origem mistas. 68

Figura 28: Concentrações de PM10 na estação Centro

comparados aos padrões de qualidade do ar (CONAMA e OMS).

71

Figura 29: Concentrações de PM10 obtidas na estação Seropédica

comparadas aos padrões de qualidade do ar (CONAMA e OMS).

Figura 30: Concentrações de PM10 na estação Duque de Caxias 
comparados aos padrões de qualidade do ar. (CONAMA e OMS).

Figura 31: Concentrações de PM10 em todos os pontos de amostragem. estações.

Figura 32: Concentrações médias $\left(\mu \mathrm{g} \mathrm{m}^{-3}\right)$ de COSA nas regiões estudadas.

Figura 33: Correlação das concentrações de sulfato de origem

não marinha (nss- $\mathrm{SO}^{2-}$ ) com a concentração de nitrato $\left(\mathrm{NO}_{3}{ }^{-}\right)$ para cada região.

Figura 34: Porcentagem dos metais extraídos na fase aquosa e ácida. 83

Figura 35: Metais majoritários da fração ácida por região.

Figura 36: Metais traços da fração ácida por região.

85

Figura 37: Concentrações médias da primavera/verão e outono/inverno. 89 Figura 38: Imagem da trajetória de massas de ar de origem mista do dia 12/05/2009.

Figura 39: Imagem da trajetória de massas de ar de origem mista do dia 03/09/09.

Figura 40: Imagens de satélites (TOM's) do dia

03 de setembro de 2009.

Figura 41: Porcentagem média das espécies em relação

as origens das trajetórias de massas de ar.

Figura 42: Porcentagem das espécies no Período

Sazonal Úmido e do Período Sazonal Seco

Figura 43: Porcentagem das espécies no Período Sazonal

Úmido e do Período Sazonal Seco da região de Seropédica.

Figura 44: Porcentagem das espécies no Período Sazonal 
Úmido e do Período Sazonal Seco da região Centro.

Figura 45: Porcentagem das espécies no Período Sazonal

Úmido e do Período Sazonal Seco da região Duque de Caxias.

99

Figura 46: Gráfico dos fatores.

101

Figura 47: Gráfico do fator 3 versus fator 1 dos componentes

principais que melhor explicam este modelo.

106

Figura 48: Discriminação das regiões onde (D) Duque de Caxias,

(C) Centro e (S) Seropédica.

Figura 49: Discriminação das correntes de massas de ar

onde (M) Marítima, (C) Continental e (I) Mista.

108 


\section{Lista de tabelas}

Tabela 1: Poluentes monitorados, suas origens e efeitos á saúde. $\quad 27$

Tabela 2: Padrões Nacionais e Internacionais da qualidade do ar. $\quad 30$

Tabela 3: Condições utilizadas para análise dos extratos

aquosos por Cromatógrafo de íons (Metrohm).

52

Tabela 4: Valores de linearidade $\left(R^{2}\right)$ e limites de quantificação

obtida de ânions por cromatografia iônica.

Tabela 5: Média e desvio padrão dos brancos dos filtros

analisados por cromatografia de íons.

54

Tabela 6: Condições do ICP-MS usadas neste trabalho.

56

Tabela 7: Parâmetros analíticos obtidos para análise de metais

Pela técnica de ICP-MS: $R^{2}$ (coeficientes de linearidade), LD (limite de detecção) e LQ (limite de quantificação).

Tabela 8: Condições do ICP OES usadas neste trabalho.

Tabela 9: Parâmetros analíticos obtidos para técnica de ICP OES:

$\mathrm{R}^{2}$ (coeficientes de linearidade), LD (limite de detecção) e

LQ (limite de quantificação).

60

Tabela 10: Condições do TOC-V CPH/CPN usadas neste trabalho.

Tabela 11: Parâmetros analíticos obtidos para técnica de (TOC): $\mathrm{R}^{2}$ (coeficientes de linearidade), LD (limite de detecção) e LQ (limite de quantificação).

Tabela 12: Concentrações médias e o desvio padrão de dispersão

de resultados (DP), valores máximos e mínimos $\left(\mu \mathrm{g} \mathrm{m}^{-3}\right)$

para PM10 obtidos em cada região. 
Tabela 13: Valores médios, desvio padrão de dispersão dos resultados (DP), mínimos e máximos da composição química dos extratos aquosos para região de Seropédica.

Tabela 14: Valores médios, desvio padrão de dispersão dos resultados (DP), mínimos e máximos da composição química dos extratos aquosos para região Centro. Tabela 15: Valores médios, desvio padrão de dispersão dos resultados (DP), mínimos e máximos da composição química dos extratos aquosos para região de Duque de Caxias.

Tabela 16: Comparação das concentrações $\left(\mu \mathrm{g} \mathrm{m}^{-3}\right)$ dos íons majoritários encontrados neste trabalho e outras localidades do Brasil.

Tabela 17: Valores médios e desvio padrão de dispersão dos resultados da composição química dos extratos ácidos para região Duque de Caxias, Centro e Seropédica.

Tabela 18: Concentrações médias e desvio padrão de dispersão dos resultados (DP) de metais comparados com os limites estabelecidos nas legislações vigentes.

Tabela 19: Correlações entre os metais da estação

Seropédica.

Tabela 20: Correlações entre os metais da estação Centro.

Tabela 21: Correlações entre os metais da estação Duque Caxias.

Tabela 22: Comparação das concentrações $\left(\mu \mathrm{g} \mathrm{m}^{-3}\right)$ dos

metais majoritários encontrados neste trabalho e outras localidades do Brasil. 
Tabela 23: Correlação da concentração de $\mathrm{Na}^{+} \mathrm{e}$

$\mathrm{Cl}^{-}$de acordo com a variabilidade temporal.

Tabela 24: Valores médios e desvio padrão de dispersão

dos resultados em $\mu_{\mathrm{g} \mathrm{m}} \mathrm{m}^{-3}$ para as regiões, nos dias $12 \mathrm{de}$

maio de 2009 e 03 de setembro de 2009.

93

Tabela 25: Análise descritiva após exclusão da

espécie formiato.

100

Tabela 26: Fatores autovalores e variância.

Tabela 27: Resultados analíticos dos parâmetros

referentes ao fator 1 .

Tabela 28: Variáveis principais e de maior

carga para cada fator.

104

Tabela 29: Resultados analíticos dos parâmetros

referentes ao fator 2 .

Tabela 30: Concentrações $\left(\mu \mathrm{g} \mathrm{m}^{-3}\right)$ diárias para as

espécies químicas presentes nos extratos aquosos da

região de Duque de Caxias.

124

Tabela 31: Concentrações $\left(\mu \mathrm{g} \mathrm{m}^{-3}\right)$ diárias para as

espécies químicas presentes nos extratos aquosos da

região de Duque de Caxias.

Tabela 32: Concentrações $\left(\mu \mathrm{g} \mathrm{m}^{-3}\right)$ diárias para

as espécies químicas presentes nos extratos aquosos

da região Centro.

Tabela 33: Concentrações $\left(\mu \mathrm{g} \mathrm{m}^{-3}\right)$ diárias para as

espécies químicas presentes nos extratos aquosos

da região Centro. 
Tabela 34: Concentrações $\left(\mu \mathrm{g} \mathrm{m}^{-3}\right)$ diárias para as espécies químicas presentes nos extratos aquosos da região Seropédica.

Tabela 35: Concentrações $\left(\mu \mathrm{g} \mathrm{m}^{-3}\right)$ diárias para as espécies químicas presentes nos extratos aquosos da região Seropédica.

Tabela 36: Concentrações $\left(\mu \mathrm{g} \mathrm{m}^{-3}\right)$ diárias para as espécies químicas presentes nos extratos ácidos da região de Duque de Caxias.

Tabela 37: Concentrações $\left(\mu \mathrm{g} \mathrm{m}^{-3}\right)$ diárias para as espécies químicas presentes nos extratos ácidos da região Centro. Tabela 38: Concentrações $\left(\mu \mathrm{g} \mathrm{m}^{-3}\right)$ diárias para as espécies químicas presentes nos extratos ácidos da região Seropédica. 


\section{Lista de abreviaturas}

ABNT Associação Brasileira de Normas Técnicas

ACP Análise dos componentes principais

CE Comunidade Européia.

CETESB Companhia de Tecnologia de Saneamento Ambiental de São Paulo

$\mathrm{Cl}$ Cromatografia de íons

CONAMA Conselho Nacional do Meio Ambiente

COSA Carbono orgânico solúvel em água

DP Desvio Padrão

IC Carbono inorgânico

ICP MS Espectrometria de massa com plasma acoplado indutivamente

ICPOES Espectrometria de emissão óptica com plasma acoplado indutivamente

INEA Instituto Estadual do Ambiente

INMET Instituto Nacional de Metrologia

LD Limite de Detecção

LQ Limite de Quantificação

MAA Média aritmética anual

MP Material particulado

OMS Organização Mundial de Saúde

PM10 Material Particulado de diâmetro inferior a10um

PSS Período Sazonal Seco

PSU Período Sazonal Úmido

RMRJ Região Metropolitana do Rio de Janeiro

TOC Carbono orgânico total

US EPA Agência Nacional de Proteção Ambiental dos Estados Unidos

WHO World Health Organization 